\title{
Analysis of Visualization Technology of 3D Spatial Geographic Information System
}

\author{
Jiudong Yang $\mathbb{D}^{1,2}$ Fenghua Wu, ${ }^{1}$ Erlong Lai, ${ }^{1,2}$ Mingyue Liu, ${ }^{1}$ Bo Liu, ${ }^{2}$ and Yingchao Zhao ${ }^{2}$ \\ ${ }^{1}$ College of Mining Engineering, North China University of Science and Technology, Tangshan, Hebei 063210, China \\ ${ }^{2}$ Hebei Tongtai Surveying and Mapping Service Co.,Ltd., Tangshan, Hebei 063200, China \\ Correspondence should be addressed to Jiudong Yang; yangjd@ncst.edu.cn
}

Received 7 April 2021; Revised 23 April 2021; Accepted 6 May 2021; Published 30 May 2021

Academic Editor: Fazlullah Khan

Copyright ( 2021 Jiudong Yang et al. This is an open access article distributed under the Creative Commons Attribution License, which permits unrestricted use, distribution, and reproduction in any medium, provided the original work is properly cited.

Traditional urban planning is generally expressed in a two-dimensional geographic information system, but its performance is limited to the plane direction. It cannot give people more natural feelings and visionary experiences. The rapid development of three-dimensional geographic information systems brings people geographic information. The three-dimensional intuitive experience, but the traditional three-dimensional geographic information system has the disadvantages that the spatial properties are incompatible, the image rendering speed is slow, and the visualization effect is poor. In this paper, the traditional domainoriented processing method is improved in spatial data processing and modeling. An optimized object-oriented optimization algorithm is proposed. The three-dimensional geographic information is optimized based on a dynamic multiresolution model and multilevel detail processing technology. The rendering of the system enhances the visualization. Based on the optimization algorithm of data processing and visualization technology proposed in this paper, the spatial data processing platform GISdata of 3D GIS is designed in this paper. At the same time, the 3D GIS is visualized based on OpenGL visualization software. It is shown that the optimization algorithm proposed in this paper has excellent preexperimental effects.

\section{Introduction}

The three-dimensional (3D) geographic information systems (GIS) is a broad subject, mainly involving many disciplines such as computing technology, cartography, surveying, and mapping. The visualization technology of 3D GIS is the application commercialization technology of 3D GIS. Many fields require 3D GIS visualization technology, such as urban construction, power, transportation construction, etc. To make the visualization technology of 3D GIS better and provide better and more convenient human beings services, further optimization and development of the technology have become very important [1-3].

Compared with the two-dimensional geographic information system, the three-dimensional geographic information system is more complicated in modeling as it comprehensively applies various modern technologies, and its advantages are also obvious [4]. At present, the application technology of 3D GIS still has problems such as large- scale modeling difficulty, unreasonable existing data processing algorithms, and poor 3D display effect [5]. Therefore, it is necessary to carry out large-scale data processing and modeling based on the existing map database and the existing data of various 2D geographic information systems. At the same time, optimize the visualization technology of 3D GIS and improve the rendering speed. Moreover, the rendering effect has become a hot and challenging point of its research [6].

The application technology of 3D GIS is mainly subject to its data acquisition and processing technology and visualization technology [7]. The traditional data processing technology is based on CAD to model three-dimensional objects and then use the data conversion algorithm to build a three-dimensional database. However, this method will bring a considerable workload for large-scale three-dimensional architecture because numerous activities cannot be automated and waste many resources. At the same time, the accuracy is poor and cannot meet the requirements [8]. 
The traditional algorithm in data representation is based on volume unit coding technology. However, this method is only widely used in two-dimensional information systems. There are still immature phenomena in three dimensions [9]. The traditional visualization technology is primarily not giving proper attention to the details and textures. It results in poor visual image effects and is not conducive to highlighting the advantages of a 3D geographic information system [10]. The main contribution of this paper is given below:

This paper optimizes and improves the disadvantages of data processing and visualization based on 3D GIS visualization technology.

This paper proposes an object-oriented data optimization processing algorithm and establishes a GIS data processing platform.

This paper proposes a dynamic multiresolution model and multilevel detail processing techniques. Taking the school as the modeling object, the experimental results based on this paper's algorithm show apparent advantages in data processing and visualization.

The sections of this paper are arranged as follows. The second section introduces the data processing technology and visualization technology of 3D GIS. The third section focuses on the proposed optimized data processing technology and visualization technology. The fourth section is mainly the experimental results and analysis of this paper. The fifth section summarizes the paper.

\subsection{GIS Technology}

2.1. Three-Dimensional Data Processing and Modeling of Geographic Information Systems. Three dimensions of $\mathrm{X}$ represent the three-dimensional spatial data, $\mathrm{Y}$ and $\mathrm{Z}$. Compared with the two-dimensional geographic information system. However, only one dimension is added; the amount of information generated is geometrically increasing. The existing data space models mainly include domainbased, object-based, and hybrid models. In essence, they are representation methods of actual objects. The more complex the objects are, the more significant the corresponding data amount and the project's complexity are. The specific principles of the three data models are as follows.

2.1.1. The Object-Based Data Processing Scheme. The main principle is to divide the information space where the model is located. The classified objects are typically analyzed and described. The actual model can be represented as shown in the following set 1 , a discrete set that can be analyzed and identified. The corresponding diagram is shown in Figure 1. The model subdivides the entire geospatial into multiple object-based collections, and the corresponding data is used as the characteristics of the object:

$$
O=\left\{O_{i}: 1 \leq i \leq n\right\} .
$$

The corresponding objects are processed in different types of spaces. The two types of dynamic operations include

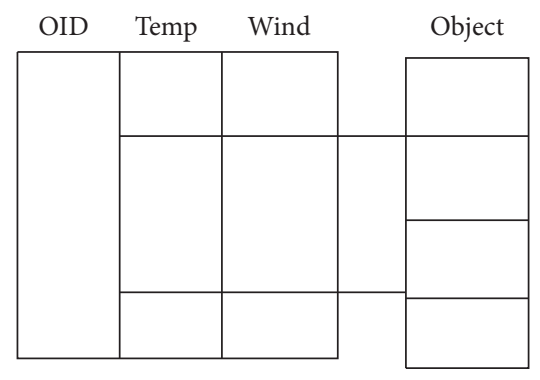

Figure 1: Object-based data model.

the appearance, deletion, and update of objects. The static operation includes the collection and the distance calculation processing in the European space and space's merging [11]. The boundary representation and structure representation are usually used to deal with spatial entities with information in different spaces. In boundary representation, a three-dimensional entity can pass through the vertices on the boundary. Lines and faces are presented. Simultaneously, the topological information and geometric information in the spatial entity are stored separately. The most significant benefit of this method is that it can fully express the entity's geometric features. However, the data relationship is complex and requires a lot of storage space. On the other hand, accuracy also needs to be further improved. The structure representation is based primarily on basic geometric constructions such as spheres, cylinders, and the like. It needs to use the regular Boolean operation to construct the three-dimensional structure of the geometry. The main advantage is that the amount of data to be processed is small, and the precision is high. However, the algorithm is complex, and the topological relationship between the expressed spatial entities is not clear [12].

In terms of modeling, the object-based data model has a low ability to express entities. There are three main options:

(1) The 1-wire box representation is divided explicitly into a wireframe model. A surface model is mainly based on many straight lines, arcs, and points to describe the contour of the three-dimensional entity.

(2) The three-dimensional formal data structure, which is mainly extended based on the two-dimensional data model, its key four essential elements: nodes, arcs, edges, and faces are the original data of the entity and other geometric relationships between the entities. These four factors determine the type of object.

(3) The solid model, its commonly used representation method, is boundary representation, mainly based on the object's boundary. The basic definition of the boundary is used to describe the geometric shape of the entity. The core idea is based on the fact that the object's boundary is composed of finite elements. In the definition of surface, each element surface has a boundary, and the line segment defines the boundary.

2.1.2. Domain-Based Model. Spatial structure architecture and domain functions are essential elements that make up a domain-based model. The domain function is essentially a 
mapping function that maps the three-dimensional spatial architecture framework to the attribute domain. The spatial structure framework divides a given area based on the distribution of spatial locations. Simultaneously, the divided subareas are referred to as bits, and the points are used to represent the position. The specific expression method is shown in the following formula 2, where $Y$ is represented as an attribute domain and $X$ is represented as a structural framework of space. Figure 2 is a domain-based model in which the model consists of two domains.

$$
F=\left\{f_{1}, f_{2} \ldots \ldots f_{n}\right\} \text {. }
$$

The data representation method of the commonly used domain-based model is the spatial segmentation method, essentially collecting body features. However, the method's geometry does not contain topology information, so the accuracy of its description is affected by the amount of data. Besides, this method requires coding and commonly used methods such as three-dimensional running coding and octree coding.

2.1.3. Mixed Data Model. The hybrid data model integrates these two data models based on the above two domain-based and object-based data models. It combines the strengths of the two data models. However, this method still has a practical test, which only stays at the theoretical level. At the same time, integration is still not possible in the processing of its database.

\subsection{Geographic Information System Visualization Technology.} After the 3D GIS data processing, establishing a compelling display image is the value of its application. In realizing $3 \mathrm{D}$ GIS visualization technology, it is necessary to combine computer graphics and other related computer-aided technologies. The application model of $3 \mathrm{D}$ visualization technology is shown in Figure 3.

Visualization technology uses visual tools to describe and present image symbols and large amounts of data. It requires $3 \mathrm{D}$ display technology. The content involved mainly includes image transformation, elimination, and corresponding color setting and shadow processing, and lighting treatment.

2.2.1. Coordinates. The essential element of a $3 \mathrm{D}$ image is the establishment of coordinates. The coordinates commonly used according to different perspectives are scene coordinates, viewpoint coordinates, and screen coordinates. The corresponding coordinates are shown in Figure 4, where the scene coordinates are established. Above the object's shape, the viewpoint coordinates are based on the origin as the viewpoint, which is necessary to complete the framing transformation. The screen coordinates are obtained from the perspective coordinate system. The primary purpose is to display the scene from a given viewpoint for accurate display. The resulting perspective effect, Figure 5, is the actual perspective effect.

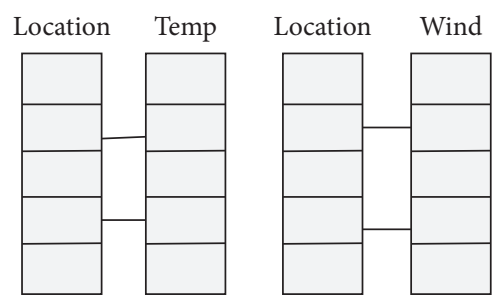

Figure 2: Domain-based model representation.

\subsubsection{D Geometric Transformation and Projection} Transformation. After establishing a standard coordinate system, the coordinates need to be geometrically transformed and displayed in a two-dimensional coordinate system. The main transformation methods include rotation, folding, translation, and transformation ratio. The conversion of the viewpoint coordinates is required before the projection transformation, as shown in Figure 6, the transformation diagram of the object coordinates to the viewpoint coordinates.

The converted image can be expressed by the following equation:

$$
\left[\begin{array}{llll}
x^{\prime} & y^{\prime} & z^{\prime} & 1
\end{array}\right]=\left[\begin{array}{llll}
x & y & z & 1
\end{array}\right] T
$$

where $T$ is the transformation matrix; taking translation as an example, the corresponding matrix when performing the corresponding operation is as follows:

$$
T=\left[\begin{array}{rrrr}
1 & 0 & 0 & 0 \\
0 & 1 & 0 & 0 \\
0 & 0 & 1 & 0 \\
T_{x} & T_{y} & T_{z} & 1
\end{array}\right] .
$$

After coordinate transformation, projection processing is required. There are two ways of corresponding projection processing: parallel projection and perspective projection. Here, parallel projection is taken as an example. If the angle between the projection direction and the projection surface is $a$, then any point $(\mathrm{x}, \mathrm{y}, \mathrm{z})$. The parallel projection is $(X, Y)$, and the equation is as follows:

$$
\left(\frac{z-z_{s}}{-1}\right)=\left(\frac{x-x_{s}}{l \cos a}\right)=\left(\frac{y-y_{s}}{l \sin a}\right), \quad z_{s}=0 .
$$

The corresponding projection image is shown in Figure 7.

2.2.3. 3D Scene Creation and Stereo Display. The establishment of the three-dimensional scene is mainly to solve the display problem of the solid model of the three-dimensional reconstruction in the three-dimensional scene. The data it needs includes raster data as well as vector data. The steps to organizing a 3D solid object into a 3D real scene are shown in Figure 8 below.

The stereoscopic display of the three-dimensional image is mainly based on OpenGL, which requires multicache technology. It needs to use a graphics card with a video stereo function to display the left and suitable landscapes on 


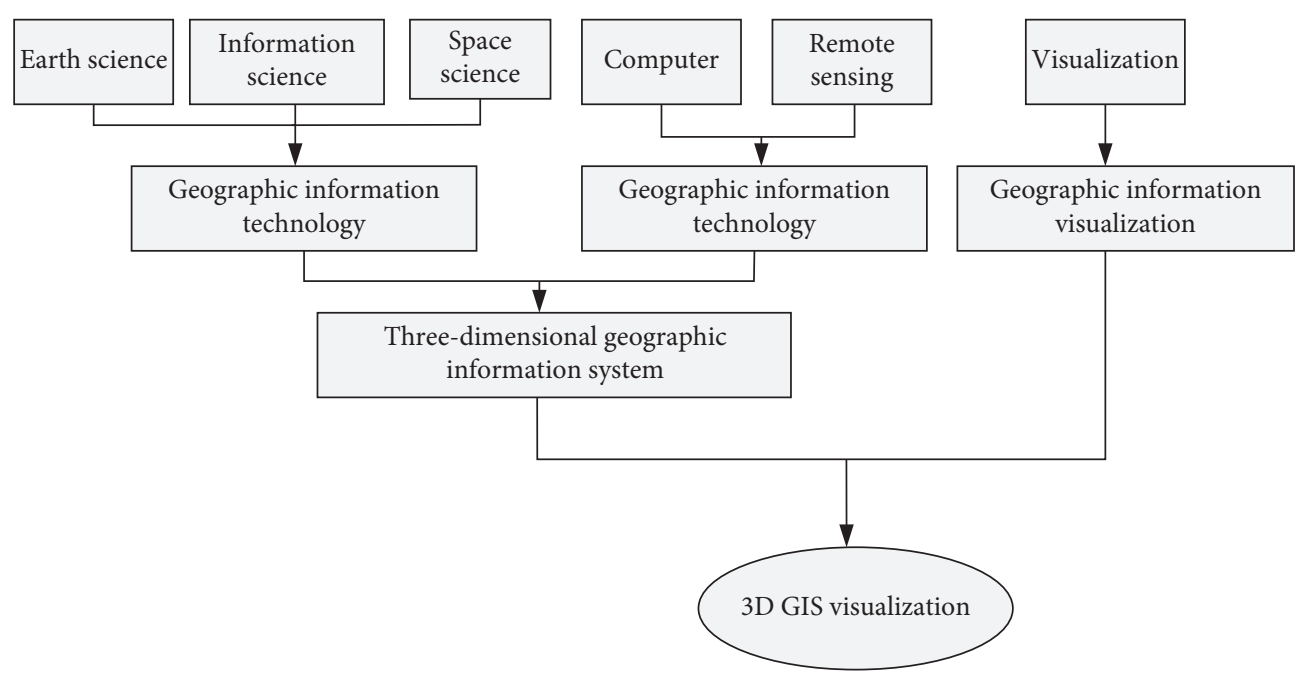

Figure 3: 3D GIS visualization system model.

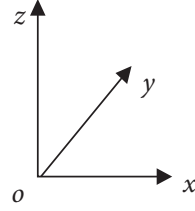

(a)

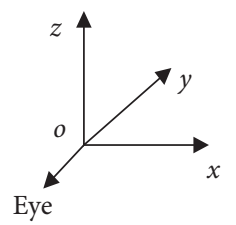

(b)
FIGURE 4: 3D GIS visualization system model (scene coordinates and viewpoint coordinates).

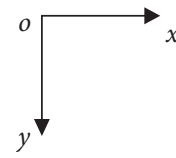

(a)

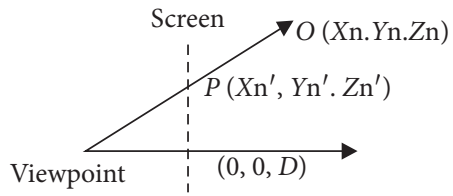

(b)
FIGURE 5: 3D GIS visualization system model (screen coordinates and perspective transformation).

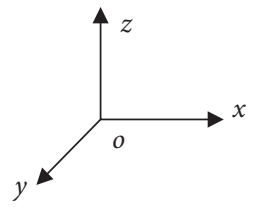

(a)

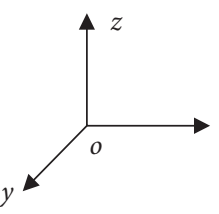

(b)
FIGURE 6: Object coordinates and viewpoint coordinates.

display at a particular refresh frequency. In addition, video stereo glasses are required to receive video stereo signals. To enhance the sense of realism, images also require shading and texture processing.

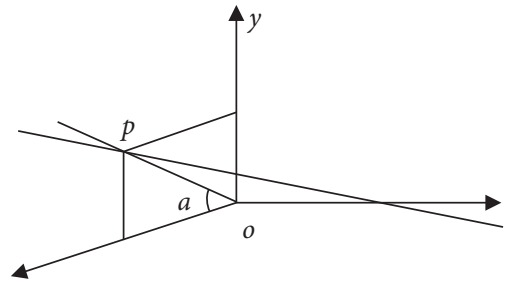

FIgURE 7: Oblique parallel projection under the coordinate observation system.

\section{Goal-Oriented Optimized Data Processing Technology and Visualization Technology}

3.1. Target-Oriented Optimization Spatial Data Processing Technology. This paper proposes a goal-oriented optimized data processing algorithm based on the data processing malpractices of the domain and the object's 3D geographic information system based on the multiple advantages of data storage, visualization, and high data analysis rate.

The data processing technology of the object-oriented $3 \mathrm{D}$ geographic information system is to use object-oriented thinking to describe the logical organization of the natural world to connect. The core idea is to abstract the entities of the objective world into the target objects of the research. The basic principle is that "any object is the target object." The target object in the algorithm is broad, which can be a set of information and a description of the corresponding operation. Interoperability between target objects is accomplished by sending messages and executing messages, where the messages are interfaces between objects. Strictly speaking, in object-oriented data processing techniques, any attribute of an entity must be represented as a variable and a set of messages for the corresponding object. In the objectoriented spatial data model, a spatial object is the spatial data and attribute data of a geographic entity and some operations and functions defined on the data. Under this idea, any 


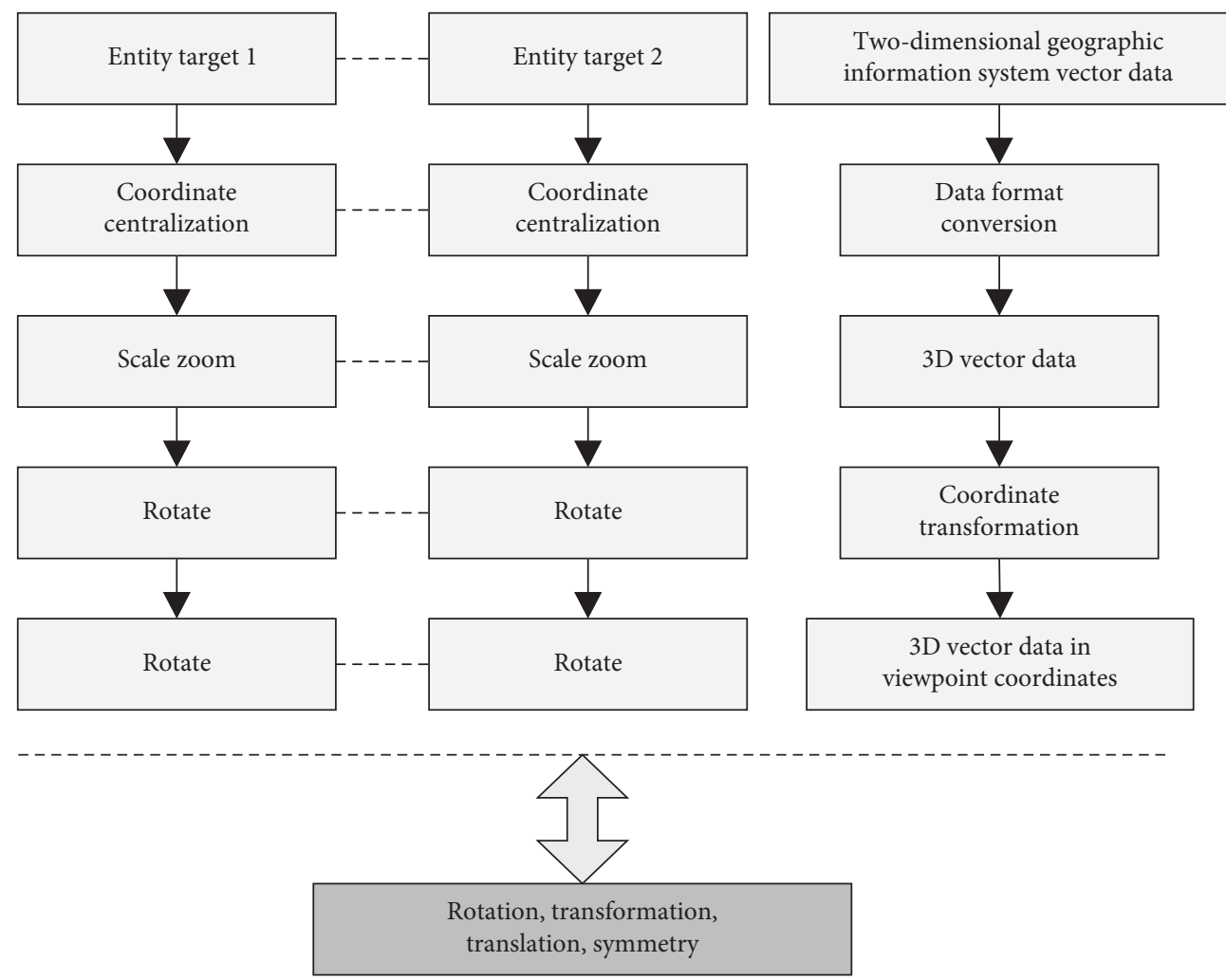

FIGURE 8: 3D solid object display.

spatial object can be derived or aggregated through basic spatial objects.

The abstract part of the model in the data processing algorithm proposed in this paper is mainly based on basic spatial objects: nodes, line segments, and triangles. The corresponding image is shown in Figure 9. Its corresponding abstract description is shown in Figure 10. Geometry and abstract attributes are two abstract classes that derive subclasses of nodes, line segments, and color textures. Each has its behavior and information, such as basic rotation, symmetry, etc., and its integration. The essential characteristics of the abstract base class. Based on the above three classes, a combination of faces and volume models can be formed. so as to carry out accurate three-dimensional modeling of the complex world. Therefore, the drawbacks of the traditional domain-based and objectbased data models are solved, and the advantages and disadvantages are complemented.

In the data storage of the optimized data processing algorithm proposed in this paper, based on the Oracle database for storage, its most significant advantage lies in supporting object target data types, support for large targets, and support for collection types and reference types. Based on the above principles, this paper designs a data management platform GISdata for a target-oriented 3D geographic information system, considering the three-dimensional application of different fields in data organization. There are significant differences in modeling and 3D analysis. The data processing platform of the 3D GIS proposed in this paper has excellent openness in architecture, and its function plug-ins have good dynamic scalability, as shown in Figure 11 below.
Its overall architecture is divided into two levels, the primary platform and the build platform. The underlying platform is three-dimensional data management and a primary display platform built on the existing GIS platform kernel module. It includes essential data management tools and basic display driver tools. The designed platform is open, i.e., an extensible 3D development platform that provides professional modeling, analysis, and visualization tools.

The advantages of the data management platform based on the data processing optimization technology of the threedimensional geographic information system proposed in this paper are as follows:

(1) Provide modeling, analysis, and visualization tools for different geographic situations.

(2) With a large amount of spatial data storage capacity, it can cope with complex model situations.

(3) Support visual 3D rendering software OpenGL, which is conducive to improving development efficiency.

3.2. Optimized Visualization Technology. After modeling and processing the spatial entities, the entities need to be inserted into a specific environment for placement and rendering to achieve realistic visual purposes. In this paper, the dynamic multiobjective model and multilevel detail processing technology are used to visualize the modeled entities and display based on software OpenGL software. Its three-dimensional display process is shown in Figure 12. 


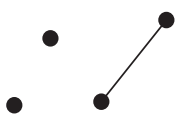

(a) (b)

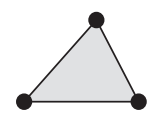

(c)

Figure 9: Three types of basic spatial objects. (a) Node, (b) line segment, and (c) triangle.

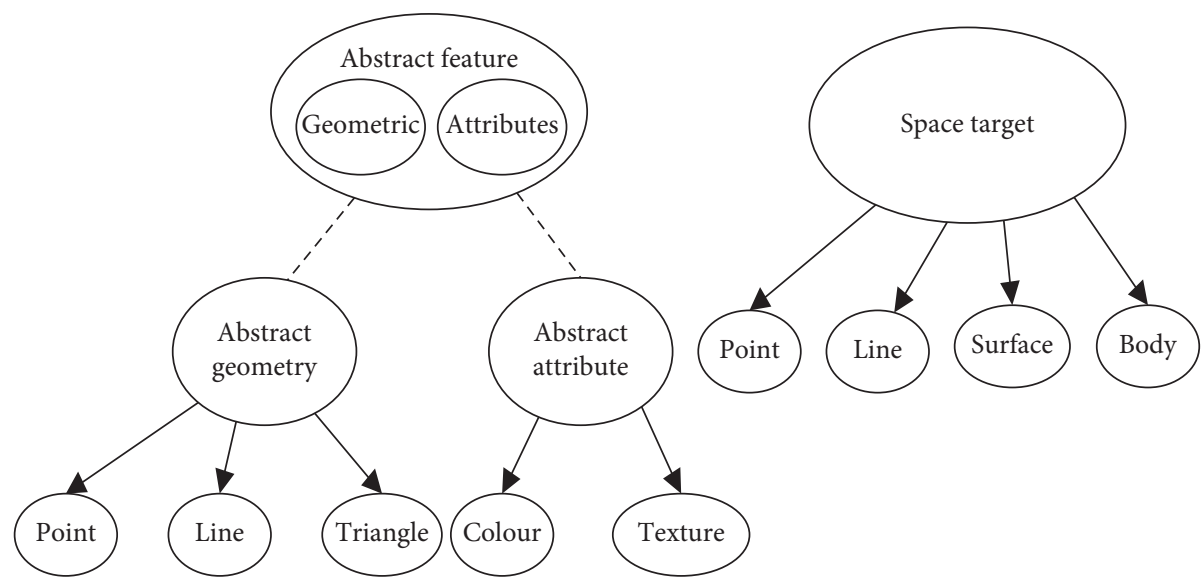

FIgURE 10: Abstract description of space target.

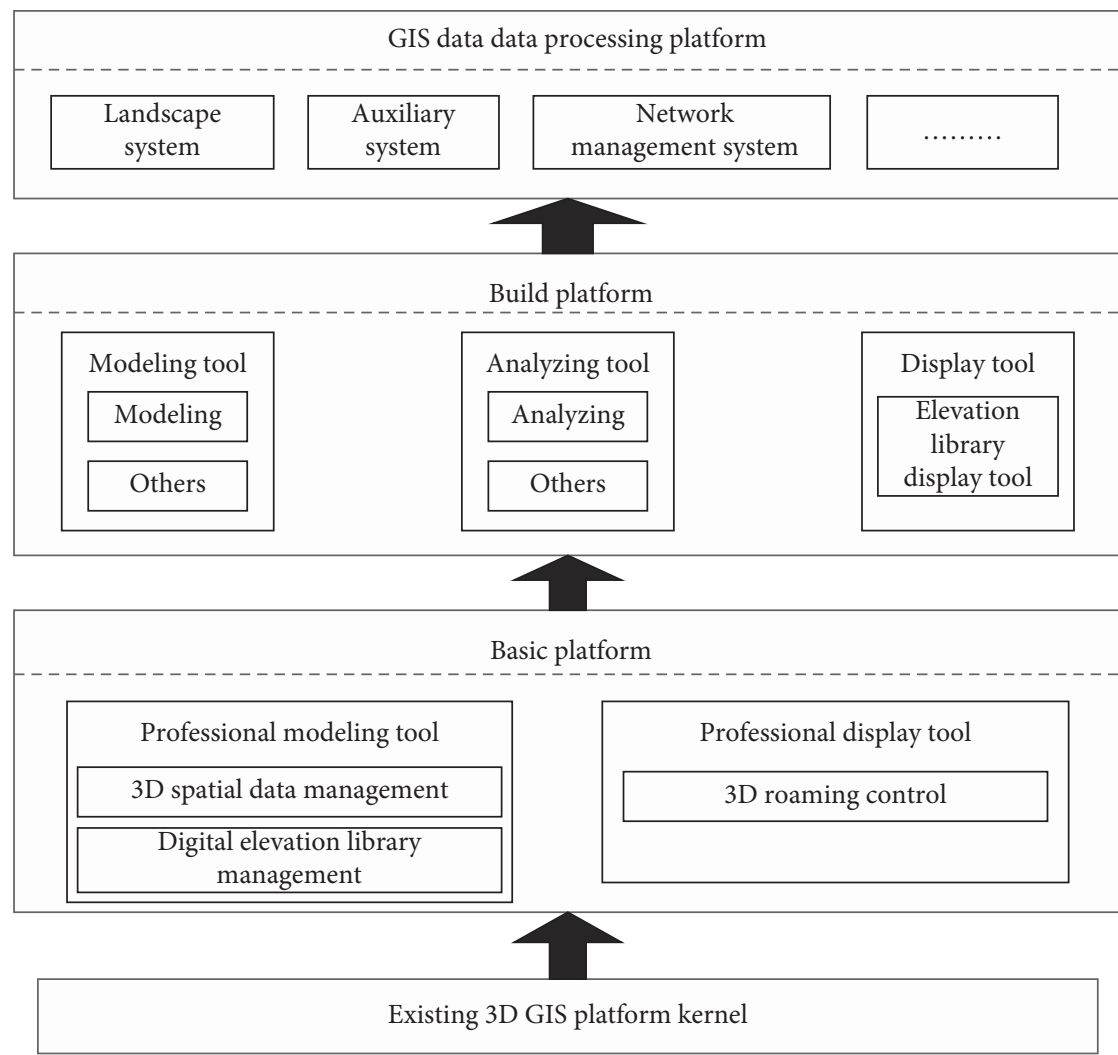

FIGURE 11: A diagrammatic architectural representation of 3D GIS data processing platform.

3.2.1. Dynamic Multiresolution Model. The dynamic multiresolution model proposed in this paper is mainly based on the information of the object such as geometric properties, texture, surface properties, and edge properties to analyze and shape the entity. A simplified model of the object at the corresponding resolution is selected according to the size of the area covered by the object on the screen. The graphic effect obtained under a given viewpoint matches the effect drawn by the most accurate model, thereby significantly improving the efficiency. The most important algorithm in the dynamic 


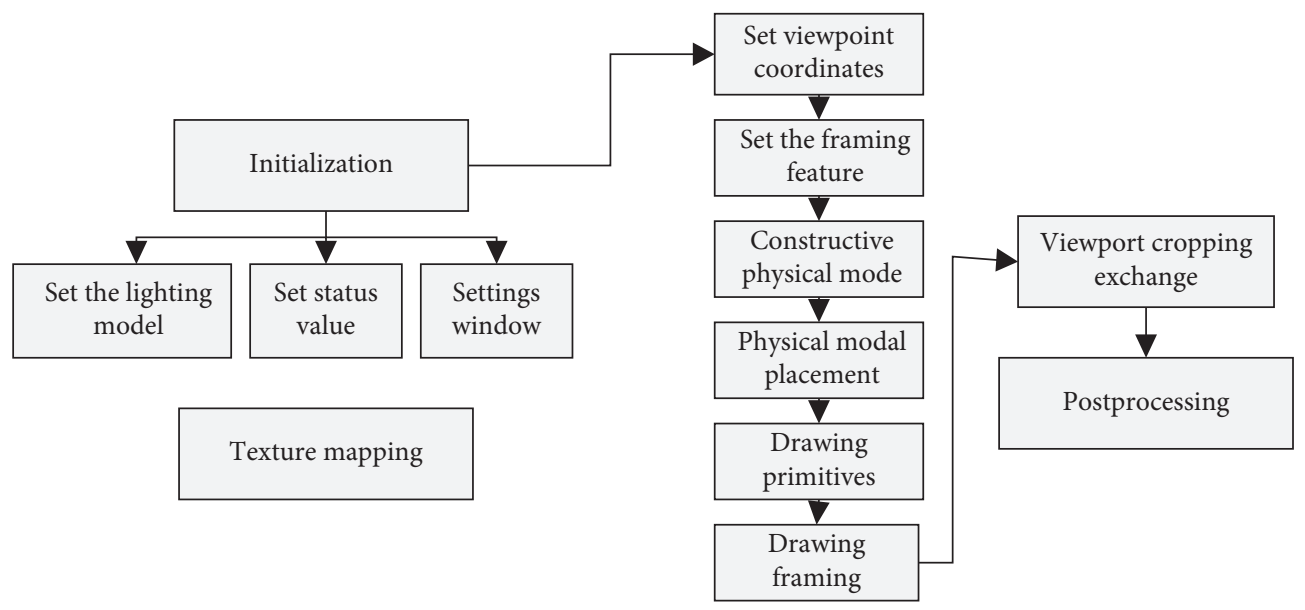

Figure 12: 3D display process.

multiresolution model is the multiresolution texture mapping algorithm divided into two-dimensional texture, three-dimensional texture, and geometric texture. The three-dimensional texture and geometric texture are stored and managed as process-defined images. The two-dimensional texture is stored and managed in the form of data files or image files. The principle of texture mapping is as shown in Figure 13. Assume that the size of a geometric region in the object space is $h$ and the distance from the viewpoint is $d$. The distance from the viewpoint to the viewpoint is $f$. The size of the region projected onto the projection surface is $n$.

3.2.2. Multilevel Detail Processing Technology. In detail processing, this paper proposes a multilevel detail processing scheme, which is a geometric description that uses different precisions for an object according to different displays. The higher the detail of the object, the more detailed the data; the finer the description, the lower the level of detail. It justifies the traditional thinking that "the image quality is as fine as possible." However, it hopes to use human visual cognition and mode according to the dominant direction of the line of sight. The line of sight is the distance in the scene from the viewpoint and the size of the projection area. Factors determine the level of detail that the scene should choose. In the proposed scheme, it is necessary to reduce the geometric complexity of the scene by simplifying the surface details of the scenes. It is worth mentioning here that simplification is performed one by one without simplifying the visual effects to solve the disadvantages of the enormous computational complexity of the method.

\section{Case Experiment Results and Analysis}

4.1. Target-Oriented Spatial Data Processing Platform. This paper designs a target-oriented spatial data processing platform for GIS data based on an object-oriented data

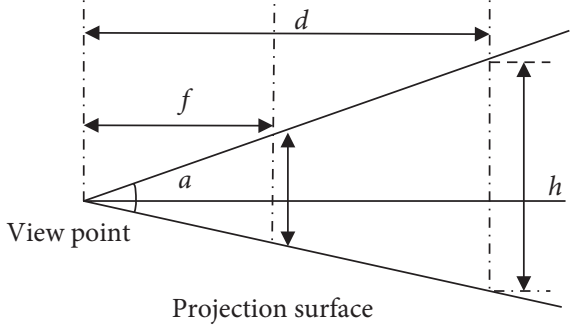

Figure 13: Projection surface and viewpoint map.

processing algorithm and its specific system functions are shown in Figure 14. Its main functions are data preparation, object editing, terrain object mapping, effect generation, and $3 \mathrm{D}$ roaming engine.

The texture projection technique based on multilevel detail processing technology is compared with the unified resolution model. The comparison table is shown in Table 1 below.

It can be seen from the table that the time consumption of multiresolution texture mapping is much less timeconsuming than the mapping of the unified resolution, which embodies the advantages of the algorithm proposed in this paper.

4.2. School-Based 3D GIS Visualization Experiment. Based on the optimization algorithm proposed in this paper, the school data model built in 3DMAX is imported into OpenGL. The translation and rotation of the model are realized in OpenGL. The corresponding three-dimensional image of the teaching building is shown in Figure 15.

The rendered road and parking lot are shown in Figure 16. 


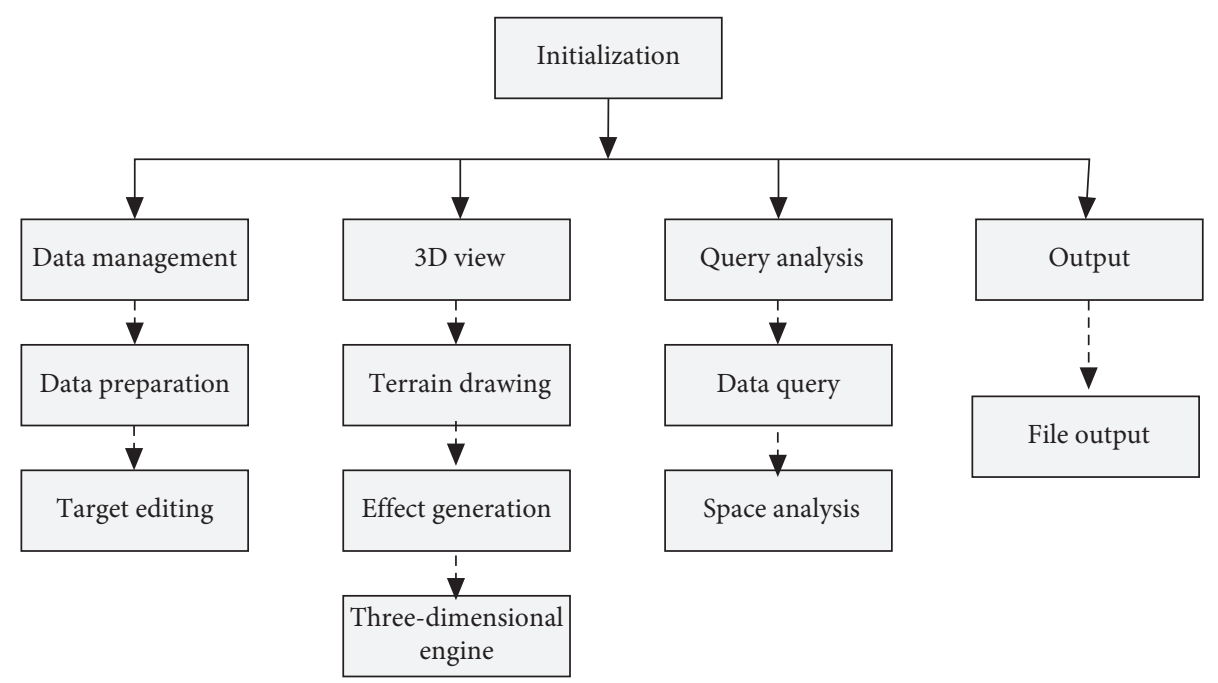

Figure 14: GISdata system functions.

TABLE 1: Case analysis comparison.

\begin{tabular}{lccccc}
\hline \multirow{2}{*}{ Original image size } & \multicolumn{2}{c}{ Subblock size } & Unified resolution model \\
& $256 * 256$ & $512 * 512$ & $64 * 64$ & $128 * 128$ & $19.12 \mathrm{~s}$ \\
$7000 * 5400$ & $19.01 \mathrm{~s}$ & $18.98 \mathrm{~s}$ & $18.66 \mathrm{~s}$ & $17.67 \mathrm{~s}$ & $4.65 \mathrm{~s}$ \\
$2048 * 2028$ & $2.5 \mathrm{~s}$ & $2.61 \mathrm{~s}$ & $2.71 \mathrm{~s}$ & $2.89 \mathrm{~s}$ & $0.8 \mathrm{~s}$ \\
$1024 * 1024$ & $0.78 \mathrm{~s}$ & $0.81 \mathrm{~s}$ & $0.85 \mathrm{~s}$ & $0.95 \mathrm{~s}$ & \multirow{2}{*}{. } \\
\hline
\end{tabular}

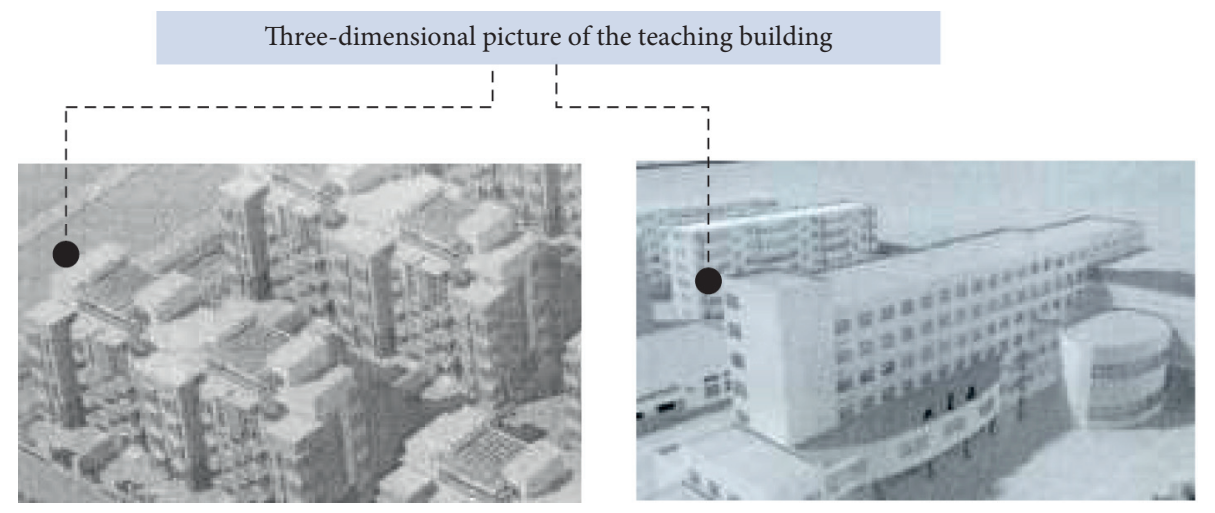

FIGURE 15: Three-dimensional picture of the teaching building based on the algorithm of this paper.

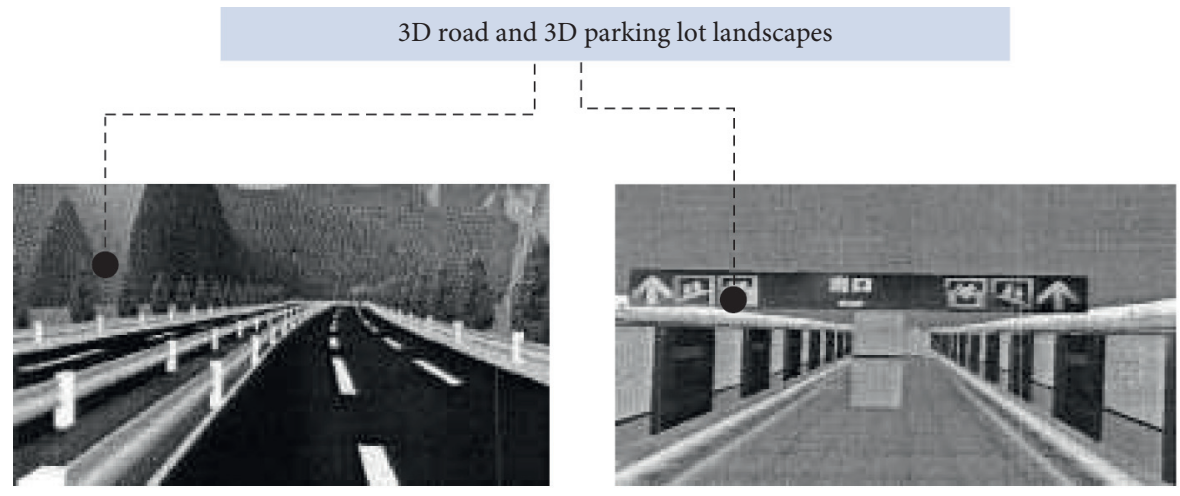

FIGURE 16: The proposed algorithm in the rendering of 3D roads and 3D parking lots. 


\section{Conclusion}

The data processing optimization algorithm and visual optimization algorithm of the three-dimensional geographic information system proposed in this paper show the disadvantages of the traditional algorithm being inefficient in data processing and poor visual rendering. The optimization algorithm based on this algorithm is designed. The school's goal-oriented data processing platform GISdata has been visualized as a geographical processing object to obtain a better visual effect. In summary, the three-dimensional geographic information system visualization algorithm proposed in this paper has excellent advantages in data processing and visualization.

\section{Data Availability}

The datasets used and/or analyzed during the current study are available from the corresponding author upon reasonable request.

\section{Conflicts of Interest}

The authors declare that they have no conflicts of interest regarding the publication of this paper.

\section{Acknowledgments}

The authors thank the National Natural Science Foundation of China (Invasiveness and Adaptability Research of Alien S. alterniflora Based on Remote Sensing Derived Functional Traits (no. 41901375).

\section{References}

[1] C. K. Cheung and W. Shi, "Measuring uncertainty of spatial features in a three-dimensional geographical information system based upon numerical analysis," Annals of GIS, vol. 7, no. 2, pp. 124-130, 2001.

[2] J. H. Gan and T. L. He, "A kind of fog visualization methods in three-dimensional geographic information system," in Proceedings of the International Symposium on Information Science and Engineering, pp. 142-144, Shanghai, China, July 2011.

[3] A. N. Kuznetsov and M. S. Syzdykov, "Evaluation of spatial brucellosis distribution using the Geographic Information System: towards building a high-performance spatial epidemiology system for supervision on zoonotic infections," in Proceedings of the International Conference on Application of Information and Communication Technologies, pp. 1-3, IEEE, Udaipur, India, October 2015.

[4] W. Shi, B. Yang, and Q. Li, "An object-oriented data model for complex objects in three-dimensional geographical information systems," International Journal of Geographical Information Science, vol. 17, no. 5, pp. 411-430, 2003.

[5] S.Y. Wan, E. L. Ritman, and W. E. Higgins, "Multigenerational analysis and visualization of large 3D vascular images," Proceedings of SPIE-The International Society for Optical Engineering, vol. 4322, no. 2, pp. 766-775, 2001.

[6] Y.-Q. Song, L. Niu, L. He, and R. Wang, "A grid-based graph data model for pedestrian route analysis in a micro-spatial environment," International Journal of Automation and Computing, vol. 13, no. 3, pp. 296-304, 2016.

[7] G. Lan, Y. Jiang, Y. Yang et al., "Research of 3D representation theory and technology of geographic information," in Proceedings of the Computer Science and Information Engineering, 2009 WRI World Congress on IEEE, pp. 326-330, Washington, DC, USA, March 2009.

[8] F. C. Ahmed and S. P. Sekar, "Using three-dimensional volumetric analysis in everyday urban planning processes," Applied Spatial Analysis and Policy, vol. 8, no. 4, pp. 393-408, 2015.

[9] W. Li, Y. L. Cai, and W. F. Lu, "A streaming technology of 3D design and manufacturing visualization information sharing for cloud-based collaborative systems," Springer Series in Advanced Manufacturing, Springer, Berlin, Germany, pp. 137-163, 2013.

[10] H. Su, Y. Wen, C. Min et al., "VGEs-oriented multi-sourced heterogeneous spatial data integration," Journal of Geo-Information Science, vol. 7146, no. 3, pp. 292-298, 2009.

[11] D. Edler, O. Huber, C. Knust, M. F. Buchroithner, and F. Dickmann, "Spreading map information over different depth layers-an improvement for map-reading efficiency?" Cartographica: The International Journal for Geographic Information and Geovisualization, vol. 49, no. 3, pp. 153-163, 2014.

[12] M. Czerwinski, S. Dumais, G. Robertson et al., "Visualizing implicit queries for information management and retrieval," in Proceedings of the Sigchi Conference on Human Factors in Computing Systems. ACM, pp. 560-567, Pittsburgh, PA, USA, May 1999. 